\title{
Editorial
}

\section{Controlling Antimicrobial-Resistant Pathogens}

\author{
William R. Jarvis, MD
}

Controlling current and emerging antimicrobialresistant pathogens has become one of the most important, yet difficult and controversial, challenges in healthcare epidemiology today. During the past two decades, we have seen the emergence and establishment of endemnicity of methicillin-resistant Staphylococcus aureus (MRSA), vancomycin-resistant enterococci (VRE), and a variety of other antimicrobial-resistant pathogens in many, if not most, hospitals in the United States. Of particular concern, during the past several years, we have witnessed the emergence of vancomycin-intermediate resistant $S$. aureus and, in 2002, the emergence of vancomycin-resistant $S$. aureus. ${ }^{1-3}$ These events should have mobilized the infection control and infectious diseases communities to apply all possible control measures to turn the tide on these costly antimicrobialresistant pathogens, but debate continues and little administrative resolve has focused on this urgent and present problem.

In most hospitals worldwide, MRSA accounts for $50 \%$ to $75 \%$ of the $S$. aureus causing hospital-acquired infections. ${ }^{4,5}$ Because of these high rates of MRSA, many infection control personnel have been reluctant to aggressively apply measures that have been repeatedly documented to control the spread of MRSA in healthcare settings, including active surveillance cultures and barrier precautions such as contact isolation. ${ }^{6}$ In the United States, VRE has increased from $0 \%$ to more than $25 \%$ of all hospital-acquired enterococcal infections among patients in intensive care units. ${ }^{5}$ VRE initially emerged at East Coast healthcare facilities, but infection control personnel in many West Coast facilities failed to learn from their East Coast colleagues. They did not introduce control measures and thus witnessed a similar establishment of endemnicity of VRE in their facilities. ${ }^{7,8}$ Both MRSA and VRE have repeatedly been documented to cause increased length of hospitaliza- tion, increased morbidity, increased mortality, and increased costs. In addition, these pathogens cause large numbers of lawsuits, driving up insurance and healthcare costs. Despite these data, debate continues. Some suggest that controlling antimicrobial use will be more effective than preventing the spread of antimicrobial-resistant pathogens. Vocal opponents of enhancing infection control measures demand more data (particularly randomized, controlled trials), even as more and more patients become colonized, become infected, and die with these pathogens. If prevention is primary, action is imperative.

In this issue of Infection Control and Hospital Epidemiology, five studies add data to this debate. In the first, Stiefel et al. assess the impact of substituting beta-lactam/beta-lactamase inhibitor combinations such as piperacillin/tazobactam or ampicillin/sulbactam (ie, antimicrobials with potent anti-anaerobic effect) for third-generation cephalosporins (ie, antimicrobials with minimal anti-anaerobic effect) on gastrointestinal flora and subsequent gastrointestinal colonization with VRE when challenged. ${ }^{9}$ Several reports have suggested that switching from third-generation cephalosporins to piperacillin/ tazobactam might reduce the emergence of VRE. When mice were treated by Stiefel et al., it was found that the impact of antimicrobial exposure occurred not only during receipt of the agent, but also for days after the exposure. Thus, although piperacillin/tazobactam inhibited VRE gastrointestinal colonization during receipt, it led to enhanced risk of VRE colonization after therapy was halted. Stiefel et al. alert us to the fact that although a variety of studies have assessed the impact of antimicrobials (especially those with potent or minimal anti-anaerobic activity) in animal models, we still have relatively little understanding of both the immediate and the long-term impact of the antimicrobials that we give to our patients on subsequent risk of col- 
onization with antimicrobial-resistant pathogens. This is especially true for high-risk patients, who receive prolonged multiple antimicrobial therapy. Stiefel et al. suggest that the adverse impact of antimicrobial therapy may persist for days, weeks, or even months in humans. This study should encourage renewed emphasis on the judicious use of all antimicrobial drugs (including some previously thought to be "safe to use" in large amounts).

A second study by the same authors expands on these findings by assessing the impact of increasing use of beta-lactamase inhibitors on the rates of VRE colonization or infection at four hospitals in the Cleveland area. ${ }^{10}$ Overall, the incidence of VRE increased during the study period at these four hospitals. During the period with increased use of agents with enhanced anti-anaerobic activity, the incidence of VRE decreased slightly at only one of the four hospitals, which appeared to have had an outbreak with very high rates of VRE (approximately 17 VRE per 10,000 patient-days of care). The lack of reduction in VRE at the other three hospitals led the authors to question again the reliability of switching to piperacillin/tazobactam for controlling VRE infections.

In the third study, Christiansen et al. describe their experience controlling and eradicating a VRE epidemic at their hospital in Australia, introduced by a nephrology patient in their intensive care unit. ${ }^{11}$ Interestingly, this hospital had previously implemented "vigorous screening and control measures" for MRSA; using this approach, the infection control community had prevented the establishment of MRSA endemnicity at all hospitals in Western Australia. When VRE was introduced in one hospital, the hospital management found this to be "unacceptable" because it seemed inconsistent with "good clinical governance." Unfortunately, administrators at U.S. hospitals have rarely shown such a strong commitment to patient safety. After the initial introduction by the index case, surveillance cultures of contacts of the index case identified three additional previously unknown VRE-colonized patients. A VRE executive group (including the chief executive of the hospital) was formed, surveillance cultures for VRE were performed for all patients on admission to and discharge from the hospital, contact isolation and hand hygiene were reinforced, antimicrobial use was modified by halting the use of third-generation cephalosporins and emphasizing the use of piperacillin/tazobactam, and environmental cultures were performed to assess the adequacy of cleaning. Additional surveillance cultures, performed from July to September 2001, identified $169 \mathrm{VRE}$-colonized patients on 23 different wards. Patients were cohorted onto VRE-colonized/infected or non-VRE-colonized/infected wards. VRE transmission was interrupted at this hospital within 6 months and eradicated during the succeeding 12 months, except for one cluster of 5 patients associated with the readmission of a previously cleared patient. Although the switch in antimicrobials may have been helpful, it seems likely that identifying colonized patients and preventing clonal spread were very important, as emphasized by the authors.
When this intervention was described at a major infectious disease meeting in the United States, the authors were criticized; those not in favor of controlling antimicrobial-resistant pathogens using enhanced infection control practices commented that the cost $(\$ 2.7$ million Australian or approximately $\$ 1.5$ million U.S.) was too great and that this task force was thus misguided in attempting to eradicate or control VRE transmission. The authors could have replied that the amount of money routinely required by U.S. hospitals to care for large numbers of MRSA- or VREinfected patients dwarfs the amount spent to eradicate MRSA and VRE at this hospital in Australia. This Australian hospital has virtually no MRSA- or VRE-infected patients in contrast to the large numbers of such patients in U.S. hospitals, where evidence-based measures to control these pathogens have been neglected. With antimicrobial-resistant pathogens, we can either pay to control them now or pay forever for not controlling them. Is having more than $50 \%$ of hospital-acquired infections being caused by methicillin-resistant strains of $S$. aureus and $25 \%$ of enterococcal infections caused by vancomycin-resistant strains acceptable? Is it consistent with "good clinical governance"? Why should patients have to accept the enormous and still expanding burden of excessive hospital stays, use of more expensive antimicrobials, greater morbidity, increased cost, and excess mortality associated with MRSA and VRE infections?

Furthermore, if one examines the approach taken by Christiansen et al., it is possible that several cost-saving measures might have been used. First, after the initial point-prevalence survey, active surveillance cultures of patients might have been focused on those at increased risk, rather than on all patients admitted to the hospital. This approach (targeted active surveillance cultures) has been used by others to control both MRSA and VRE transmission. ${ }^{12,13}$ Second, a large proportion of the cost was related to the extensive and frequent environmental cultures. Focusing the environmental cultures to those really necessary (ie, to establish that terminal cleaning is adequate) and to monitor the continued adequacy of environmental cleaning would have saved a lot of money. Nevertheless, rather than being criticized for this successful intervention, these authors are to be congratulated for putting the phrase "prevention is primary" into action and eradicating an important antimicrobial-resistant pathogen.

In the fourth study, Hachem et al. evaluate the impact of stool VRE surveillance cultures and contact isolation on controlling clonal VRE transmission among patients with hematologic malignancy in a hospital not implementing other new infection control measures or antimicrobial control measures. ${ }^{14}$ During a 4-year period, VRE bloodstream infections decreased 8-fold (from 32 to 4 VRE bloodstream infections per 100,000 patient-days), despite an unchanged rate of vancomycin use. Clonal VRE transmission was terminated within 2 years. These data are similar to those from an intervention by Montecalvo et al. in which similar measures led to a significant reduction in both VRE colonization and infection rates on an oncology 
ward. ${ }^{15}$ Furthermore, given the high attributable mortality and costs associated with these infections, Montecalvo et al. documented that such preventive measures were costeffective. ${ }^{16}$ Given that hematology-oncology patients are at high risk for VRE colonization or infection, those caring for such patients should seriously consider implementing such an evidence-based program to reduce the adverse impact associated with VRE in this population. ${ }^{6}$

In the fifth study, Boyce et al. review the data on using measures to control MRSA transmission and then provide data from their implementation of an intervention program to control MRSA at their facility. ${ }^{17}$ This excellent review concludes that active surveillance cultures, use of barrier precautions for MRSA-colonized or MRSA-infected patients (contact isolation), hand hygiene, and identifying and treating healthcare workers implicated in MRSA transmission have been effective in controlling the transmission of MRSA. Because of continuing transmission of MRSA despite a vigorous hand hygiene campaign and contact isolation of the occasional patient recognized to be colonized because of the results of routine clinical cultures, Boyce et al. decided to implement active surveillance cultures and contact isolation. Concerned about the logistics of trying to implement this approach hospital-wide immediately (a concern voiced by others), they started in the surgical intensive care unit. They made no other changes in infection control or antimicrobial use. In the first 5 months of the intervention, they identified 23 patients with MRSA-positive surveillance cultures; the proportion of patients who acquired MRSA in the unit decreased significantly after the intervention ( $2.2 \%$ to $0.7 \% ; P=.033)$. Fifty-one percent of those with MRSA were detected only by the surveillance cultures. Because of the success of this intervention in the intensive care unit, Boyce et al. then expanded the program to the hematology-oncology ward. In the first 4 months, 18 (5\%) of 350 patients admitted to that ward were found to be colonized with MRSA ( $56 \%$ would have been detected only by surveillance cultures). After the intervention, only 3 (0.9\%) of the admitted patients acquired MRSA on the hematology-oncology ward. These data resemble those of Hachem et al., illustrating protection of hematology-oncology patients. ${ }^{14,15}$

Recently, the Society for Healthcare Epidemiology of America (SHEA) took a bold stand for patient safety by recommending a comprehensive program to control antimicrobial-resistant pathogens, including MRSA and VRE. ${ }^{6}$ This program includes active surveillance cultures to identify the colonized reservoir, barrier precautions (contact isolation) for colonized and infected patients, routine hand hygiene, and judicious use of antimicrobials. The SHEA guideline documents many studies using active surveillance cultures in which the transmission of MRSA, VRE, or both was markedly reduced or eliminated. Recently, a mathematical model was published by Perencevich et al. showing that active surveillance cultures for VRE were necessary for effective control in intensive care units. ${ }^{18}$ They concluded that standard precautions and isolation of the occasional patient recognized to be colonized through the results of routine clinical cultures are minimally effective. The 2004 SHEA annual meeting included 10 to 15 presentations documenting the impact of reducing or eliminating transmission of MRSA or VRE and improving patient outcomes. Within the next several weeks, it is anticipated that the Centers for Disease Control and Prevention (CDC) will be publishing a draft of the revised isolation guideline in the Federal Register for public comment. If it does not recommend active surveillance cultures and contact isolation as routine measures for all facilities, we must ask why. The data are overwhelming that this approach improves patient outcomes and should be strongly recommended. If prevention is primary and patient safety is a priority, then the guideline should recommend the measures illustrated by these and other articles that have been successful in controlling the transmission of antimicrobial-resistant pathogens. Twenty-five years of data show that without active surveillance cultures to identify the colonized reservoir, prevention will fail. Repeated successes associated with the use of active surveillance cultures and contact isolation are not due to chance alone. Endemic MRSA or VRE does not disappear from institutions spontaneously. The public comment period for the CDC's revised isolation guideline represents an opportunity for the infection control community to influence the final guideline. The infection control community should demand routine use of evidence-based measures for preventing spread as illustrated repeatedly in this issue of Infection Control and Hospital Epidemiology. The CDC guideline is supposed to be evidence-based. Where is the evidence that standard precautions will suddenly start controlling the spread of MRSA and VRE? Like the hospital management in the Australian hospital mentioned earlier, we shouldn't accept the current state of VRE and MRSA infections in our hospitals. It is not consistent with "good clinical governance." Patient safety should be our top priority. Active surveillance cultures will detect the unrecognized MRSA-colonized or VRE-colonized patients and help prevent transmission and infection. Isn't this the goal of infection control and healthcare epidemiology? Isn't this true promotion of healthcare quality? Do we have to wait until consumers or their lawyers demand implementation of these programs? The time to act is now.

\section{REFERENCES}

1. Smith TL, Pearson ML, Wilcox KR, et al. Emergence of vancomycin resistance in Staphylococcus aureus. N Engl J Med 1999;340:493-501.

2. Centers for Disease Control and Prevention. Staphylococcus aureus resistant to vancomycin: United States, 2002. MMWR 2002;51:565-567.

3. Centers for Disease Control and Prevention. Public Health Dispatch: vancomycin-resistant Staphylococcus aureus-Pennsylvania, 2002. MMWR 2002;51:902

4. Richet HM, Benbachir M, Brown D, et al. Are there regional variations in the diagnosis, surveillance, and control of methicillin-resistant Staphylococcus aureus? Infect Control Hosp Epidemiol 2003;24:334-342.

5. National Nosocomial Infections Surveillance (NNIS) System. National Nosocomial Infections Surveillance System report: data summary from January 1992-June 2001, issued August 2001. Am I Infect Control 2001;29:404-421.

6. Muto CA, Jernigan JA, Ostrowsky BE, et al. SHEA guideline for preventing nosocomial transmission of multidrug-resistant strains of Staphylococcus aureus and Enterococcus. Infect Control Hosp Epidemiol 2003;24:362-386.

7. Winston LG, Bangsberg DR, Chambers HF 3rd, et al. Epidemiology of 
vancomycin-resistant Enterococcus faecium under a selective isolation policy at an urban hospital. Am I Infect Control 2002;30:400-406.

8. Rosenberg J, Jarvis WR, Abbott SL, Vugia DJ for the California Emerging Infections Program. Emergence of vancomycin-resistant enterococci in San Francisco Bay area hospitals during 1994 to 1998. Infect Control Hosp Epidemiol 2004;25:408-412.

9. Stiefel U, Pultz NJ, Helfand MS, Donskey CJ. Increased susceptibility to vancomycin-resistant Enterococcus intestinal colonization persists after completion of anti-anaerobic antibiotic treatment in mice. Infect Control Hosp Epidemiol 2004;25:373-379.

10. Stiefel U, Paterson DL, Pultz NJ, Gordon SM, Aron DC, Donskey CJ. Effect of the increasing use of piperacillin/tazobactam on the incidence of vancomycin-resistant enterococci in four academic medical centers. Infect Control Hosp Epidemiol 2004;25:380-383.

11. Christiansen KJ, Tibbett PA, Beresford W, et al. Eradication of a large outbreak of a single strain of vanB vancomycin-resistant Enterococcus faecium at a major Australian teaching hospital. Infect Control Hosp Epidemiol 2004;25:384-390.

12. Byers KE, Anglim AM, Anneski CJ, et al. A hospital epidemic of vancomycin-resistant Enterococcus: risk factors and control. Infect Control Hosp Epidemiol 2001;22:140-147.
13. Ostrowsky BE, Trick WE, Sohn A, et al. Control of vancomycin-resistant enterococcus colonization in healthcare facilities in a region. $N$ Engl J Med 2001;344:1427-1433.

14. Hachem R, Graviss L, Hanna $\mathrm{H}$, et al. Impact of surveillance for vancomycin-resistant enterococci on controlling a bloodstream outbreak among patients with hematologic malignancy. Infect Control Hosp Epidemiol 2004;25:391-394.

15. Montecalvo MA, Jarvis WR, Unan J, et al. Infection-control measures reduce transmission of vancomycin-resistant enterococci in an endemic setting. Ann Intern Med 1999;131:269-272.

16. Montecalvo MA Jarvis WR. Uman J, et al. Costs and savings associated with infection control measures that reduced transmission of vancomycin-resistant enterococci in an endemic setting. Infect Control Hosp Epidemiol 2001;22:437-442.

17. Boyce JM, Havill NL, Kohan C, Dumigan DG, Ligi CE. Do infection control measures work for methicillin-resistant Staphylococcus aureus? Infect Control Hosp Epidemiol 2004;25:395-401.

18. Perencevich EN, Fishman DN, Lipsitch M, Harris AD, Morris JG, Smith D. Projected benefits of active surveillance for vancomycin-resistant enterococci in ICU settings. Clin Infect Dis 2004;38:1108-1115. 